\title{
Markers of cardiovascular autonomic dysfunction predict COPD in middle-aged subjects
}

\author{
Fabrizio Ricci, ${ }^{1,2}$, Per Wollmer ${ }^{3,4}$, Gunnar Engström¹, Artur Fedorowski ${ }^{1,5}$ and \\ Viktor Hamrefors ${ }^{1,6}$
}

\begin{abstract}
Affiliations: ${ }^{1}$ Dept of Clinical Sciences, Lund University, Malmö, Sweden. ${ }^{2}$ Institute for Advanced Biomedical Technologies, Dept of Neuroscience, Imaging and Clinical Sciences, "G.d'Annunzio" University, Chieti, Italy. ${ }^{3}$ Dept of Translational Medicine, Lund University, Malmö, Sweden. ${ }^{4}$ Dept of Medical Imaging and Physiology, Skåne University Hospital, Malmö, Sweden. ${ }^{5}$ Dept of Cardiology, Skåne University Hospital, Malmö, Sweden. ${ }^{6}$ Dept of Internal Medicine, Skåne University Hospital, Malmö, Sweden.
\end{abstract}

Correspondence: Viktor Hamrefors, Clinical Research Centre, Lund University, Jan Waldenströms Gata 35, Building 91, Floor 12, SE-205 02 Malmö, Sweden. E-mail: Viktor.Hamreforsamed.lu.se

@ERSpublications

Clinical markers of subtle cardiovascular autonomic dysfunction predict incident COPD in a middleaged population http://ow.ly/J93J30i0O4s

Cite this article as: Ricci F, Wollmer P, Engström G, et al. Markers of cardiovascular autonomic dysfunction predict COPD in middle-aged subjects. Eur Respir J 2018; 51: 1702481 [https://doi.org/ 10.1183/13993003.02481-2017].

ABSTRACT Autonomic dysfunction is commonly observed in chronic obstructive pulmonary disease (COPD) and may relate to the known comorbidity with coronary artery disease (CAD). We hypothesised that clinical markers of cardiovascular autonomic dysfunction predict COPD in the population, independently of CAD.

In a population-based cohort of 24768 subjects (mean age 45 years) without baseline airflow obstruction, we analysed the cross-sectional relationship of one-minute orthostatic systolic blood pressure (SBP) and diastolic blood pressure (DBP) changes and resting heart rate with forced vital capacity (FVC) and forced expiratory volume in $1 \mathrm{~s}(\mathrm{FEV} 1)$. Cox regression models were used to analyse the association of orthostatic SBP and DBP changes (SBP/DBP decrease) and resting heart rate with incident COPD over a 32-year follow-up.

Baseline orthostatic SBP decrease $(p=0.020)$ and DBP decrease $(p=0.001)$ were associated with reduced FVC, whereas resting heart rate was associated with reduced FVC and FEV1 $(p<0.001)$. After adjustment for smoking and baseline lung function, SBP decrease predicted COPD (hazard ratio (HR) 1.10 per $10 \mathrm{mmHg}$, 95\% CI 1.03-1.18). Resting heart rate predicted COPD among smokers (HR 1.11 per 10 beatsper-minute increase, 95\% CI 1.05-1.18). Results were similar in subjects without CAD.

Subtle signs of cardiovascular autonomic dysfunction may precede the development of COPD in middle-aged subjects. This association is independent of the relationship between cardiovascular autonomic dysfunction and CAD.

This article has supplementary material available from erj.ersjournals.com

Received: Nov 302017 | Accepted after revision: Jan 222018

Conflict of interest: None declared.

Support statement: This study was supported by grants from the Swedish Heart and Lung Foundation, Medical Faculty of Lund University, ALF-funds, Crafoord Foundation, Ernhold Lundströms Research Foundation, Region Skåne, and Hulda and Conrad Mossfelt Foundation. Funding information for this article has been deposited with the Crossref Funder Registry.

Copyright @ERS 2018 


\section{Introduction}

There is a strong association between chronic obstructive pulmonary disease (COPD) and cardiovascular disease (CVD) in the general population, especially between COPD and coronary artery disease (CAD) [1-4]. The link between COPD and CAD is only partially explained by known common risk factors, e.g. tobacco smoking [5-8] or other airborne pollutants [9-13]. A number of conditions, including systemic inflammation [14,15] and common genetic variants [16], have been proposed as possible mediators. However, a significant proportion of the relationship between COPD and CAD still remains elusive [3], especially in non-smokers, who represent approximately one-quarter of the COPD population [17].

In recent years, manifestations of cardiovascular autonomic dysfunction, including increased resting heart rate [18] and orthostatic hypotension $(\mathrm{OH})$ [19], have been shown to predict CVD and CAD. Furthermore, $\mathrm{OH}$ can predict mortality due to respiratory disease [20]. Given the direct involvement of the autonomic nervous system in the respiratory system and the presence of autonomic dysfunction in COPD [21], we hypothesised that markers of cardiovascular autonomic dysfunction may predict the development of COPD in the general population.

Accordingly, we aimed to test whether markers of cardiovascular autonomic dysfunction, i.e. orthostatic blood pressure (BP) instability and elevated resting heart rate, predict incident COPD in the middle-aged population without signs of airflow obstruction at baseline.

\section{Methods}

\section{Study population}

The study population consisted of 33346 inhabitants of the city of Malmö in Sweden, recruited between 1974 and 1992 for the Malmö Preventive Project (MPP). At baseline, participants were screened for hypertension, diabetes, obesity, hyperlipidaemia, smoking status, and history of cardiovascular and lung disease. Those who confirmed regular or occasional smoking in the preceding 3 months were defined as smokers. "Heavy smoking" was defined as $>20$ cigarettes per day. Physical activity was assessed as previously described [22] (detailed in the supplementary methods). All subjects fasted overnight prior to the baseline investigations but were allowed to drink water. All examinations were performed in the morning. Among numerous variables [23], anthropometric measurements, BP, resting heart rate and pulmonary function test (PFT) data were recorded and the subjects provided blood samples. The health service authority of Malmö approved and funded the screening programme. All participants provided informed consent.

For the current study, subjects with prevalent COPD $(n=126,0.4 \%)$ were excluded. PFT data were available in 28834 subjects without overt COPD (see below). To further eliminate subjects with airflow obstruction at baseline, we excluded 4066 subjects (14.1\%) with a forced expiratory volume in $1 \mathrm{~s}$ (FEV1) and forced vital capacity (FVC) below the lower limit of normal according to the Global Lung Function Initiative equations [24]. Ultimately, 24768 subjects were eligible for the study, and haemodynamic data of orthostatic systolic blood pressure (SBP) response, diastolic blood pressure (DBP) response and resting heart rate were available in 24702, 24694 and 24641 subjects, respectively (figure 1).

\section{Definitions of baseline characteristics}

$\mathrm{OH}$ was defined according to the international consensus as a decrease in SBP $\geqslant 20 \mathrm{mmHg}$ and/or a decrease in DBP $\geqslant 10 \mathrm{mmHg}$ [25]. Diabetes was defined as a fasting plasma glucose concentration $\geqslant 7.0 \mathrm{mmol} \cdot \mathrm{L}^{-1}$, current pharmacological treatment of diabetes or self-reported history of diabetes [26].

\section{Haemodynamic measurements}

BP was measured by specially trained nurses using the auscultatory method with a mercury sphygmomanometer and an appropriately sized cuff placed around the right arm supported at the heart level. The first set of two BP and two heart rate readings was taken after a 10-min rest in the supine position. Participants were then asked to stand up and the second set of two BP measurements was taken after $1 \mathrm{~min}$ in the standing position. The average values of both supine and standing haemodynamic parameters were calculated.

An orthostatic BP decrease was defined as the average supine BP minus the average standing BP: a positive value meant a BP decrease on standing (SBP and DBP decrease). Resting heart rate was recorded in the supine position as beats per minute (bpm).

\section{Pulmonary function tests}

Pulmonary function at baseline was assessed as FVC and FEV1 using a Spirotron apparatus (Drägerwerk AG, Lübeck, Germany) and carried out by trained nursing staff, without bronchodilation. One acceptable 


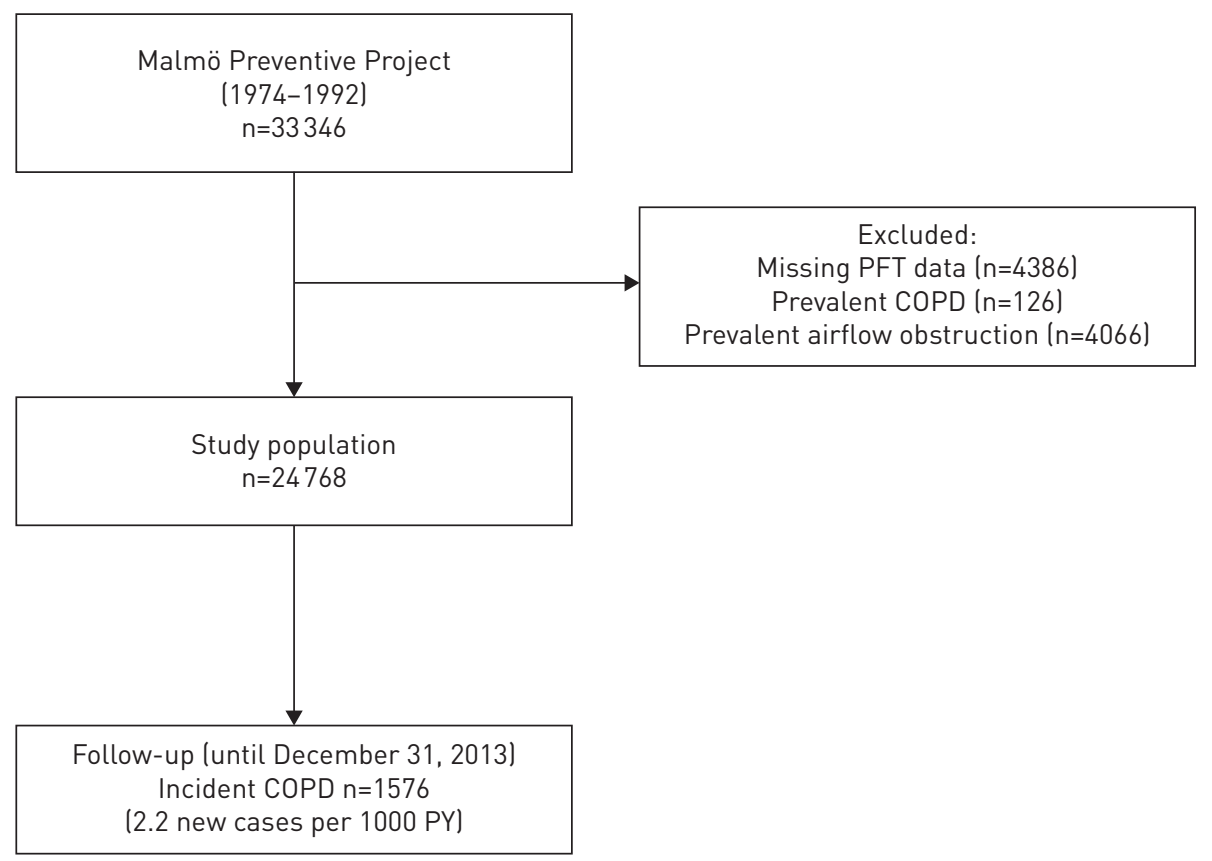

FIGURE 1 Selection process of the study population. PFT: pulmonary function test; COPD: chronic obstructive pulmonary disease; PY: person-years.

manoeuvre was required. FVC and FEV1 were standardised for age and height using the Global Lung Function Initiative equations [24].

\section{Definition and retrieval of endpoints}

The endpoints were identified through linkage of the 10-digit personal identification number of each Swedish citizen with the Swedish Patient Register, as previously described [27] and validated for COPD [28]. The subjects were followed from baseline until admission to hospital for COPD, death, emigration from Sweden or December 31, 2013, whichever came first.

CAD was defined as fatal or non-fatal myocardial infarction, death from ischaemic heart disease, coronary artery bypass grafting or percutaneous coronary intervention. In addition to the Swedish Patient Register, we used the Swedish Coronary Angiography and Angioplasty Registry, the national registers of surgical procedures and the Swedish Cause of Death Register.

We also retrieved data on hospitalisations for $\mathrm{OH}$ and syncope from the Swedish Patient Register, though this was only available to December 31, 2011.

A full description and International Classification of Diseases codes for endpoint retrieval can be found in the supplementary methods.

\section{Statistical analysis}

We assessed the cross-sectional relationship of the haemodynamic parameters with FVC and FEV1 at baseline using linear regression models, adjusted for age, sex, current smoking and predicted FVC or FEV1.

The associations between the haemodynamic parameters at baseline and incident COPD during follow-up were tested using Cox regression models. The haemodynamic parameters were entered as continuous variables; SBP decrease was also dichotomised according to the consensus definition of $\mathrm{OH}(20 \mathrm{mmHg}$ decrease) and according to tertiles of SBP decrease. We used minimally (age and sex) adjusted models, as well as multivariable-adjusted models that included the variables body mass index (BMI), current smoking, diabetes, total cholesterol, supine BP, antihypertensive therapy, and FVC and FEV1 expressed as a percentage of predicted values. Among smokers, heavy smoking $(n=2331,20.6 \%)$ was also added as a covariate.

Analyses were stratified according to four baseline factors that potentially influence the association between the haemodynamic factors and COPD: sex, smoking status defined as current versus no smoking, smoking quantity and physical inactivity. Formal interaction analyses between smoking and the 
haemodynamic parameters on incident COPD during follow-up were performed using Cox regression models including age, sex, smoking, and FVC and FEV1 expressed as a percentage of predicted values, in addition to the interaction term of smoking $\times$ haemodynamic parameter. Analyses were also stratified according to incident CAD during follow-up. Finally, we explored the incidence of COPD according to hospitalisation for syncope or symptomatic $\mathrm{OH}$ during follow-up.

The proportional hazard assumption for Cox regression analyses was tested by visually inspecting the survival curves of tertiles of the haemodynamic parameters. All analyses were performed using IBM SPSS Statistics version 24 (SPSS Inc., Chicago, IL, USA). All tests were two-sided whereby $\mathrm{p}<0.05$ was considered statistically significant.

\section{Results}

\section{Baseline characteristics}

Baseline characteristics of the study population are shown in table 1 . The subjects were followed for a median time of 32 years, during which COPD was diagnosed in 1576 subjects (6.4\%), rendering an incidence rate of 2.2 per 1000 person-years. The median time from baseline to the COPD diagnosis was 25 years.

Baseline orthostatic SBP decrease $(\beta-14 \mathrm{~mL}$ per $10 \mathrm{mmHg}, \mathrm{p}=0.020)$ and DBP decrease $(\beta-34 \mathrm{~mL}$ per $10 \mathrm{mmHg}, \mathrm{p}=0.001)$ were associated with FVC, but not $\mathrm{FEV}_{1}(\mathrm{p}=0.68$ and $\mathrm{p}=0.20)$. Resting heart rate showed linear correlations with baseline FVC $(\beta-56 \mathrm{~mL}$ per $10 \mathrm{bpm}, \mathrm{p}<0.001)$ and $\mathrm{FEV} 1(\beta-30 \mathrm{~mL}$ per 10 bpm, $\mathrm{p}<0.001)$.

\section{Orthostatic blood pressure response in relation to incident COPD}

An orthostatic SBP decrease, but not an orthostatic DBP decrease, predicted incident COPD in the minimally adjusted and the multivariable-adjusted models. An orthostatic SBP decrease according to the $\mathrm{OH}$ definition $(>20 \mathrm{mmHg}$ ) did not predict COPD (table 2). In contrast, an SBP decrease $>5 \mathrm{mmHg}$ (upper tertile of the study population) was associated with incident COPD in the multivariable-adjusted model (hazard ratio (HR) 1.142 in relation to first tertile, 95\% CI 1.007-1.295, p=0.039; figure 2).

Stratification according to baseline current smoking revealed that an orthostatic SBP decrease predicted COPD in current smokers, but not in non-smokers (table 2). There was, however, no significant interaction between current smoking and orthostatic SBP decrease on incident COPD ( $\mathrm{p}$-interaction=0.226). Adjusting for heavy smoking did not change the overall results among smokers.

TABLE 1 Baseline characteristics of the study population

\begin{tabular}{|c|c|c|c|}
\hline & All & Non-smokers & Smokers \\
\hline Subjects $\mathrm{n}$ & 24768 & 13448 & 11320 \\
\hline Age years & $44.5 \pm 7.3$ & $44.6 \pm 7.2$ & $44.3 \pm 7.4$ \\
\hline Females & 28.0 & 29.4 & 26.4 \\
\hline BMI $\mathrm{kg} \cdot \mathrm{m}^{-2}$ & $24.5 \pm 3.5$ & $24.7 \pm 3.5$ & $24.1 \pm 3.5$ \\
\hline Supine SBP mmHg & $128.6 \pm 15.4$ & $130.0 \pm 15.3$ & $126.8 \pm 15.2$ \\
\hline Supine DBP $\mathrm{mmHg}$ & $84.9 \pm 9.4$ & $86.0 \pm 9.2$ & $83.6 \pm 9.5$ \\
\hline Postural SBP decrease $\mathrm{mmHg}$ & $1.6 \pm 7.2$ & $1.5 \pm 7.1$ & $1.7 \pm 7.3$ \\
\hline Postural DBP decrease $\mathrm{mmHg}$ & $-2.4 \pm 4.4$ & $-2.5 \pm 4.4$ & $-2.2 \pm 4.5$ \\
\hline Orthostatic hypotension & 2.5 & 2.2 & 2.8 \\
\hline SBP decrease $\geqslant 20 \mathrm{mmHg}$ & 1.9 & 1.7 & 2.1 \\
\hline Resting heart rate bpm & $69.0 \pm 10.0$ & $69.2 \pm 10.1$ & $68.7 \pm 9.8$ \\
\hline FVC mL & $4169 \pm 996$ & $4265 \pm 993$ & $4054 \pm 988$ \\
\hline FVC $\%$ predicted & $89 \pm 15$ & $92 \pm 15$ & $86 \pm 15$ \\
\hline FEV1 mL & $3359 \pm 796$ & $3451 \pm 791$ & $3250 \pm 788$ \\
\hline FEV $1 \%$ predicted & $90 \pm 15$ & $93 \pm 15$ & $86 \pm 15$ \\
\hline FEV1/FVC ratio & $0.81 \pm 0.07$ & $0.81 \pm 0.07$ & $0.81 \pm 0.07$ \\
\hline Physical inactivity & 49.6 & 45.8 & 54.2 \\
\hline Antihypertensive treatment & 4.8 & 5.6 & 4.7 \\
\hline Diabetes & 4.5 & 4.4 & 4.7 \\
\hline CAD & 0.4 & 0.3 & 0.4 \\
\hline
\end{tabular}

Data are presented as mean \pm SD or $\%$ of total, unless otherwise stated. BMI: body mass index; SBP: systolic blood pressure; DBP: diastolic blood pressure; bpm: beats per min; FVC: forced vital capacity; FEV1: forced expiratory volume in $1 \mathrm{~s}$; CAD: coronary artery disease. 


\begin{tabular}{|c|c|c|c|}
\hline & Sample size \pm events & HR $(95 \% \mathrm{CI})$ per $10 \mathrm{mmHg}$ decrease & p-value \\
\hline \multicolumn{4}{|l|}{ All subjects } \\
\hline$\Delta$ SBP Model $1^{\#}$ & $24697 \pm 1569$ & $1.123(1.049-1.202)$ & 0.001 \\
\hline$\Delta$ SBP Model $2^{\pi}$ & $24647 \pm 1564$ & $1.101(1.026-1.180)$ & 0.007 \\
\hline$\Delta \mathrm{SBP}>20 \mathrm{mmHg}$ & $24647 \pm 1564$ & $1.216(0.886-1.669)$ & 0.227 \\
\hline$\triangle \mathrm{DBP}$ Model $1^{\#}$ & $24689 \pm 1569$ & $1.052(0.940-1.178)$ & 0.378 \\
\hline$\triangle \mathrm{DBP}$ Model $2^{\pi}$ & $24639 \pm 1564$ & $0.989(0.886-1.104)$ & 0.841 \\
\hline \multicolumn{4}{|l|}{ Smokers } \\
\hline$\Delta$ SBP Model $1^{\#}$ & $11280 \pm 1228$ & $1.119(1.036-1.208)$ & 0.004 \\
\hline$\triangle$ SBP Model $2^{\pi}$ & $11259 \pm 1224$ & $1.112(1.028-1.202)$ & 0.008 \\
\hline$\triangle$ SBP Model $3^{+}$ & $11259 \pm 1224$ & $1.112(1.029-1.201)$ & 0.007 \\
\hline$\Delta S B P>20 \mathrm{mmHg}$ & $11259 \pm 1224$ & $1.268(0.898-1.790)$ & 0.177 \\
\hline$\Delta$ DBP Model $1^{\#}$ & $11276 \pm 1228$ & $1.004(0.885-1.138)$ & 0.954 \\
\hline$\triangle \mathrm{DBP}$ Model $2^{\pi}$ & $11255 \pm 1224$ & $1.001(0.9882-1.136)$ & 0.983 \\
\hline$\triangle \mathrm{DBP}$ Model $3^{+}$ & $12255 \pm 1224$ & $1.008(0.889-1.143)$ & 0.901 \\
\hline \multicolumn{4}{|l|}{ Non-smokers } \\
\hline$\Delta$ SBP Model $1^{\#}$ & $13413 \pm 341$ & $1.032(0.890-1.198)$ & 0.673 \\
\hline$\triangle$ SBP Model $2^{\pi}$ & $13384 \pm 340$ & $1.056(0.906-1.231)$ & 0.485 \\
\hline$\Delta S B P>20 m m g^{\pi}$ & $13384 \pm 340$ & $0.972(0.430-2.197)$ & 0.946 \\
\hline$\Delta$ DBP Model $1^{\#}$ & $13409 \pm 341$ & $0.932(0.732-1.187)$ & 0.570 \\
\hline$\triangle \mathrm{DBP}$ Model $2^{\pi}$ & $13380 \pm 340$ & $0.934(0.728-1.198)$ & 0.589 \\
\hline
\end{tabular}

HR: hazard ratio; $\triangle \mathrm{SBP}$ : orthostatic systolic blood pressure decrease; $\triangle \mathrm{DBP}$ : orthostatic diastolic blood pressure decrease. " : model adjusted for age and sex; ": model adjusted for age, sex, body mass index, \pm current smoking, diabetes, total cholesterol, supine systolic blood pressure or supine diastolic blood pressure, antihypertensive therapy, and forced vital capacity and forced expiratory volume in $1 \mathrm{~s}$ expressed as a percentage of predicted values; ${ }^{+}$: as Model 2 but additionally adjusted for smoking quantity $>20$ cigarettes per day.

Stratification according to smoking quantity revealed that an orthostatic SBP decrease significantly predicted COPD only among the larger group of non-heavy smokers (table 3). However, there was no interaction between heavy smoking and an orthostatic SBP decrease on incident COPD.

Analyses stratified according to sex showed that an SBP decrease predicted COPD only among the larger group of men (supplementary table $\mathrm{S} 1$ ).

Adding physical inactivity to the multivariable-adjusted model did not change the overall association between orthostatic SBP decrease and incident COPD (HR 1.101 per $10 \mathrm{mmHg}$ SBP decrease, 95\% CI $1.026-1.180, \mathrm{p}=0.007)$. However, stratification according to physical inactivity revealed that an orthostatic SBP decrease predicted COPD only among physically inactive subjects (supplementary table S2). Subjects reporting physical inactivity showed a larger orthostatic SBP decrease $(1.7$ versus $1.5 \mathrm{mmHg}, \mathrm{p}=0.016)$, lower FEV1 (88\% versus $92 \%$ of predicted) and FVC ( $87 \%$ versus $91 \%$ of predicted), and were more likely to be smokers (50\% versus $42 \%, \mathrm{p}<0.001)$ and men $(76 \%$ versus $68 \%, \mathrm{p}<0.001)$.

\section{Resting heart rate in relation to incident COPD}

Resting heart rate predicted incident COPD in the multivariable-adjusted models, but not in the minimally adjusted model. This was explained by the confounding effect of smoking on resting heart rate, which was found to be higher in non-smokers $(69.2 \mathrm{bpm})$ compared with smokers $(68.7 \mathrm{bpm}, \mathrm{p}<0.001)$. Moreover, there was a significant interaction between resting heart rate and smoking on incident COPD ( $\mathrm{p}$-interaction=0.045). Accordingly, resting heart rate predicted incident COPD only in smokers (table 4). Resting heart rate predicted COPD only among the non-heavy smokers; however, there was no interaction between heavy smoking and heart rate on incident COPD (table 3).

Among smokers, resting heart rate was associated with lower FVC $(\beta-66 \mathrm{~mL}$ per $10 \mathrm{bpm}, \mathrm{p}<0.001)$ and FEV $1(\beta-38 \mathrm{~mL}$ per $10 \mathrm{bpm}, \mathrm{p}<0.001)$ in models adjusted for age, sex and individually predicted absolute values of FVC or FEV1.

As for the SBP decrease, resting heart rate predicted COPD only among the larger group of men (supplementary table S1). 


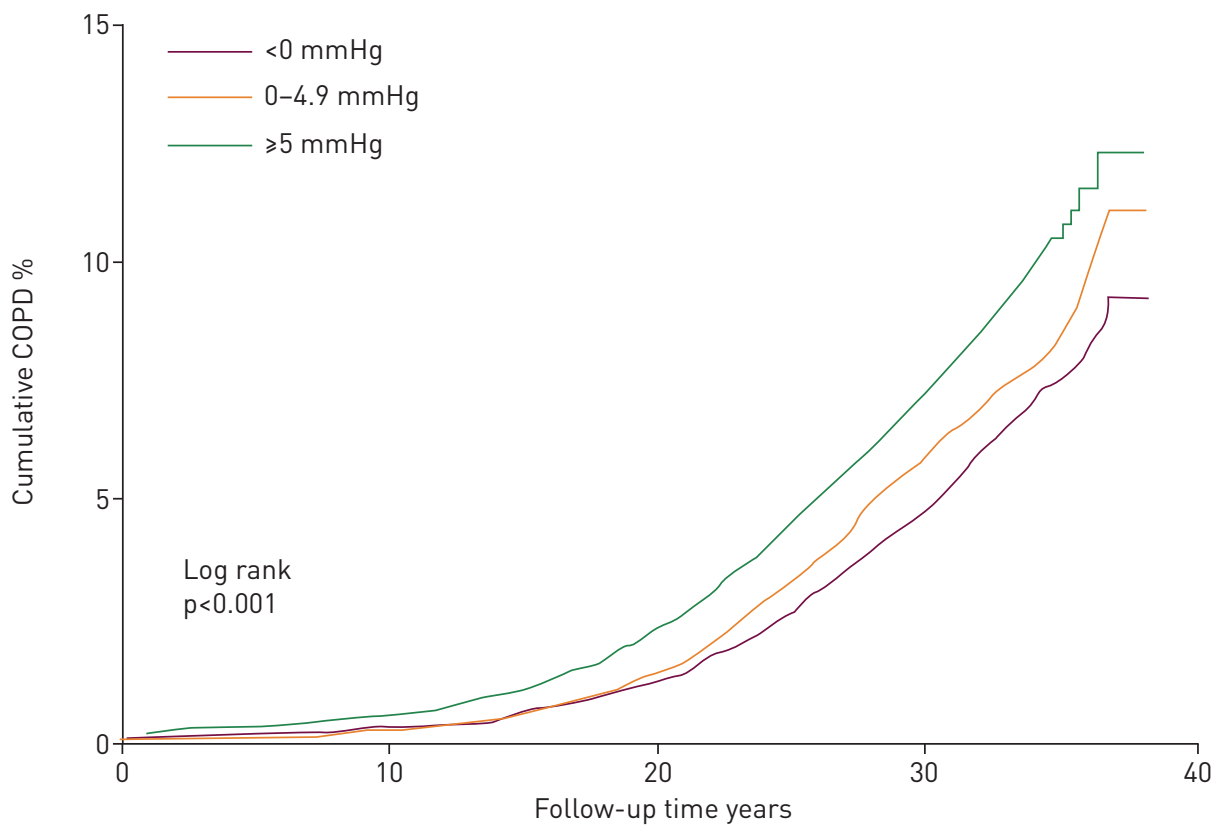

\begin{tabular}{llllll}
\multicolumn{2}{c}{ Number at risk } & & & & \\
$<0 \mathrm{mmHg}$ & 8145 & 7805 & 7121 & 4136 & 0 \\
$0-4.9 \mathrm{mmHg}$ & 7959 & 7584 & 6914 & 4633 & 0 \\
$\geqslant 5 \mathrm{mmHg}$ & 8597 & 8088 & 7091 & 4206 & 0
\end{tabular}

FIGURE 2 Incident chronic obstructive pulmonary disease (COPD) during follow-up by tertiles of orthostatic systolic blood pressure response at baseline.

Adding physical inactivity to the multivariable-adjusted model did not change the association between resting heart rate and incident COPD (HR 1.062 per $10 \mathrm{bpm}, 95 \%$ CI 1.006-1.210, p=0.029). In stratified analyses, resting heart rate did not significantly predict COPD in either physically inactive or physically active subjects (supplementary table S2).

Analyses stratified according to CAD

A total of 89 subjects $(0.4 \%)$ had CAD at baseline. During follow-up, 5000 (20.3\%) additional subjects developed CAD. In the 19679 subjects without overt CAD, an orthostatic SBP decrease predicted incident COPD, whereas resting heart rate predicted incident COPD among smokers only (table 5).

Incident COPD in relation to episodes of $\mathrm{OH}$ or syncope

A total of 707 subjects were hospitalised because of $\mathrm{OH}$ or syncope. During follow-up, 107 of them (15.1\%) were also hospitalised owing to COPD, as compared to $6.1 \%$ of those without $\mathrm{OH}$ or syncope.

TABLE 3 The relationship between baseline haemodynamic parameters and incident COPD stratified according to smoking quantity in smokers

Sample size \pm events

HR per $10 \mathrm{mmHg}$ or bpm $(95 \% \mathrm{Cl})$

p-value

\begin{tabular}{llll}
\hline$\leqslant 20$ cigarettes per day & & & \\
$\Delta$ SBP & $8777 \pm 893$ & $1.157(1.054-1.270)$ & 0.002 \\
RHR & $8749 \pm 886$ & $1.106(1.028-1.190)$ & 0.007 \\
$>\mathbf{2 0}$ cigarettes per day & & & 0.880 \\
$\Delta$ SBP & $2319 \pm 312$ & $1.013(0.858-1.196)$ & 0.173 \\
RHR & $2311 \pm 311$ & $1.084(0.966-1.216)$ &
\end{tabular}

All analyses adjusted for age, sex, body mass index, diabetes, total cholesterol, supine systolic blood pressure, antihypertensive therapy, and forced vital capacity and forced expiratory volume in $1 \mathrm{~s}$ expressed as a percentage of predicted values. $p$-interaction for smoking quantity $\times \Delta S B P$ on incident $C O P D=0.156$. p-interaction for smoking quantityxheart rate on incident COPD=0.117. COPD: chronic obstructive pulmonary disease; bpm: beats per min; HR: hazard ratio; $\triangle$ SBP: orthostatic systolic blood pressure decrease; RHR: resting heart rate. 


\begin{tabular}{|c|c|c|c|}
\hline & Sample size \pm events & HR per 10 bpm ${ }^{\#}(95 \% \mathrm{Cl})$ & p-value \\
\hline \multicolumn{4}{|l|}{ All subjects } \\
\hline Model 1 & $24636 \pm 1561$ & $1.028(0.978-1.081)$ & 0.274 \\
\hline Model $2^{+}$ & $24582 \pm 1556$ & 1.064 (1.009-1.123) & 0.023 \\
\hline Over median 68 bpm & $24582 \pm 1556$ & $1.121(1.011-1.244)$ & 0.030 \\
\hline \multicolumn{4}{|l|}{ Smokers } \\
\hline Model 1 & $11244 \pm 1220$ & $1.120(1.059-1.185)$ & $<0.001$ \\
\hline Model $2^{+}$ & $11223 \pm 1216$ & $1.111(1.045-1.181)$ & 0.001 \\
\hline Model $3^{\S}$ & $11223 \pm 1216$ & $1.112(1.046-1.182)$ & 0.001 \\
\hline Over median 67 bpm & $11223 \pm 1216$ & $1.219(1.084-1.371)$ & 0.001 \\
\hline \multicolumn{4}{|l|}{ Non-smokers } \\
\hline Model $1^{\top}$ & $13388 \pm 341$ & $0.983(0.883-1.095)$ & 0.754 \\
\hline Model $2^{+}$ & $13355 \pm 340$ & $0.944(0.842-1.060)$ & 0.331 \\
\hline Over median 68 bpm & $13355 \pm 340$ & $0.864(0.692-1.079)$ & 0.197 \\
\hline
\end{tabular}

HR: hazard ratio; bpm: beats per min. " : HRs are reported per $10 \mathrm{bpm}$ except for the dichotomous variable denoting median resting heart rate; ${ }^{\uparrow}$ : model adjusted for age and sex; $^{+}$: model adjusted for age, sex, body mass index, \pm current smoking, diabetes, total cholesterol, supine systolic blood pressure, antihypertensive therapy, and forced vital capacity and forced expiratory volume in $1 \mathrm{~s}$ expressed as a percentage of predicted values; ${ }^{\S}$ : as Model 2 but additionally adjusted for smoking quantity > 20 cigarettes per day.

Excluding the subjects with episodes of $\mathrm{OH}$ or syncope did not change the main results of SBP decrease or resting heart rate in relation to incident COPD (data not shown).

\section{Discussion}

We observed that subtle manifestations of cardiovascular autonomic dysfunction, i.e. orthostatic BP decrease and elevated resting heart rate, are significantly associated with impaired lung function and predict the development of COPD in the middle-aged population. Moreover, we show that the relationship between orthostatic BP instability and incidence of COPD is also maintained in subjects without overt CAD during follow-up.

The autonomic nervous system is directly involved in the homeostasis of the respiratory system. There is also evidence of an "inflammatory reflex", through which the nervous system is involved in the regulation of inflammation [29], a hallmark of COPD. Thus, a relationship between autonomic nervous system malfunction and COPD is quite plausible from a pathophysiological perspective. Previous research has focused on the occurrence of autonomic dysfunction in manifest COPD, in which patients demonstrate elevated resting heart rate, reduced heart rate variability, reduced baroreflex sensitivity [21] and pathologic responses to the Valsalva manoeuvre [30, 31].

TABLE 5 The relationship between baseline haemodynamic parameters and incident COPD stratified according to CAD

\begin{tabular}{lccc} 
& Sample size \pm events & HR per $\mathbf{1 0} \mathbf{~} \mathbf{m H g}$ or bpm (95\% CI) & p-value \\
\hline No CAD & & & \\
$\Delta$ SBP in all & $19579 \pm 1094$ & $1.106(1.016-1.204)$ & 0.020 \\
$\Delta$ SBP in smokers & $8471 \pm 861$ & $1.118(1.017-1.229)$ & 0.021 \\
RHR all & $19526 \pm 1086$ & $1.063(0.996-1.134)$ & 0.064 \\
RHR smokers & $8443 \pm 853$ & $1.097(1.020-1.179)$ & 0.013 \\
CAD & $5068 \pm 470$ & $1.081(0.957-1.221)$ & 0.209 \\
$\Delta$ SBP in all & $2787 \pm 363$ & $1.102(0.959-1.267)$ & 0.169 \\
$\Delta$ SBP in smokers & $5056 \pm 470$ & $1.086(0.983-1.199)$ & 0.105 \\
RHR in all & $2779 \pm 363$ & $1.149(1.026-1.285)$ & 0.016 \\
RHR in smokers & & & \\
\hline
\end{tabular}

All analyses adjusted for age, sex, body mass index, diabetes, total cholesterol, supine SBP, antihypertensive therapy, and forced vital capacity and forced expiratory volume in $1 \mathrm{~s}$ expressed as a percentage of predicted values. HR: hazard ratio; CAD: coronary artery disease; bpm: beats per min; $\triangle$ SBP: orthostatic systolic blood pressure decrease; RHR: resting heart rate. 
A postural SBP decrease was previously reported to predict increased mortality from respiratory diseases in MPP [20]. In other cohorts, COPD has been identified as a risk factor for traumatic falls [32, 33], while in elderly patients $\mathrm{OH}$ has been found to coexist with COPD [34]. Our study is the first to show that subtle signs of cardiovascular autonomic dysfunction may predict incident COPD many years in advance in relatively young and apparently healthy subjects without airflow obstruction at baseline.

Remarkably, an orthostatic BP decrease was cross-sectionally associated with reduced FVC, whereas elevated heart rate was associated with both reduced FVC and FEV1. At baseline, an orthostatic BP decrease may be related to a loss of lung capacity rather than obstruction. This can be observed in a number of conditions, including COPD, restrictive lung diseases, heart failure and diabetes [35, 36]. These conditions and their corresponding subclinical phenotypes may well coexist with subtle signs of cardiovascular autonomic dysfunction. Conversely, FEV1 is more specific to airflow obstruction [37]. A possible explanation for the absent association between an orthostatic BP decrease and FEV1 is that the subtle signs of cardiovascular dysfunction precede any decrease in FEV1 by many years.

An orthostatic SBP decrease, on a continuous scale and dichotomised as $5 \mathrm{mmHg}$, predicted incident COPD, whereas manifest $\mathrm{OH}$ [38] did not. This may be because the prevalence of $\mathrm{OH}$ at baseline (2.5\%) was rather low. Previous studies have documented that an orthostatic SBP decrease of $<5 \mathrm{mmHg}$ is representative of normal controls [39] with preserved orthostatic homeostasis. This is identical to the cut-off limit for the highest tertile of orthostatic SBP decrease in our study, suggesting that individuals in the third tertile demonstrated signs of subtle to overt autonomic dysfunction. Thus, the finding of a positive relationship between smaller magnitudes of systolic BP decreases and incident COPD emphasises that subtle and subclinical cardiovascular autonomic dysfunction may be an early predictor of increased COPD risk, especially in the presence of concomitant exposure to smoking, a major factor in the development of both COPD and COPD-related peripheral neuropathy [40]. The fact that the associations were only present among non-heavy smokers is likely a result of lack of power, given that only $20 \%$ of the smokers reported heavy smoking. Moreover, the median time from baseline to first COPD event was long (25 years), supporting the hypothesis that changes in cardiovascular autonomic dysfunction may precede the development of manifest COPD by many years. COPD was, however, much more common in subjects who were also hospitalised owing to $\mathrm{OH}$ or syncope during follow-up, which further supports a strong comorbidity and parallel development of manifest autonomic dysfunction and COPD.

The impact of elevated resting heart rate as a marker of cardiovascular autonomic dysfunction deserves special consideration. Patients with COPD demonstrate a higher resting heart rate as a central feature of autonomic dysfunction [21]. Elevated heart rate is also observed in COPD exacerbations and is a common side effect of bronchodilators [41]. We found a strong association between an elevated resting heart rate and lung function at baseline and incidence of COPD during follow-up. Unexpectedly, resting heart rate was lower among smokers than among non-smokers at baseline. Furthermore, there was an interaction between resting heart rate and smoking on the incidence of COPD during follow-up, meaning that an elevated resting heart rate predicted COPD only among smokers. Although we cannot exclude residual confounding, an elevated heart rate also predicted COPD after adjustment for baseline lung function. Thus, the effect of resting heart rate on incident COPD may go beyond the potential relationship between autonomic dysfunction and a subtle lung function impairment that was already present at baseline.

Physical activity is thought to be inversely related to autonomic function. In our study, an orthostatic SBP decrease, but not resting heart rate, seems to relate to the risk of future COPD only among physically inactive subjects. This is most likely based on a heavier risk factor burden for both autonomic dysfunction and COPD in this group; however, future studies should examine whether physical activity that improves autonomic function can also decrease COPD incidence. Obesity could influence autonomic function. Although adjustment for BMI did not affect the observed relationships, only $6.6 \%$ of the study participants were obese (i.e. $\mathrm{BMI} \geqslant 30)$. Thus, we were not adequately powered to further study how obesity might influence the relationships between autonomic function and COPD. This should be further explored in more recent cohort studies, in which the prevalence of obesity is likely to be higher.

Our results were to a large extent consistent when comparing all subjects with subjects without overt CAD during follow-up. The slight attenuation of significance for resting heart rate most likely reflects the reduced statistical power in the subgroup analysis. Even though we cannot exclude a sequential pathway through the development of subclinical atherosclerosis, we believe that our findings support the view that autonomic dysfunction is associated with COPD, independently of CAD.

Subclinical parasympathetic dysfunction has been found to positively correlate with the severity of hypoxaemia in COPD, a relationship further confirmed by the acetylcholine sweat-spot test [40, 42]. Grading of autonomic impairment can be quantitated through the composite autonomic severity score, using cardiovascular reflex tests and the quantitative sudomotor axonal reflex test in three domains: 
cardiovagal, adrenergic and sudomotor. Alternatively, autonomic impairment can be graded through the quantitative autonomic reflex and small fibres tests (QASAT), including three main modules: cardiovascular, cerebral blood flow and small fibre neuropathy [43, 44]. Further research should be fostered to test the importance in the disease process and the incremental prognostic value of the presence, site and severity of dysautonomia, as assessed by the above tests and scores, in both patients with overt COPD and those without airflow obstruction but with risk factors for COPD.

Some limitations should be addressed. First, MPP did not include a standing heart rate measurement. Second, even though the PFT data in MPP show the expected association between lung function, smoking and outcomes [27, 45], PFT in MPP was performed before the time of international standardisation, meaning that the procedure included only one acceptable manoeuver. Moreover, spirometry was performed without a bronchodilator, so we possibly excluded a number of subjects with reversible airflow obstruction who did not meet the criteria for COPD. Although such subjects cannot be considered completely healthy in terms of lung function, their exclusion may have underestimated our findings. Moreover, pre-bronchodilator PFT could explain the slightly reduced mean values of FEV1 and FVC in relation to Global Lung Function Initiative predicted values. Third, although the COPD endpoint used in the current study has been validated [28], a hospital diagnosis of COPD does not necessarily indicate a confirmed diagnosis on PFT. Fourth, although markers of cardiovascular autonomic dysfunction predicted COPD in subjects who were free from manifest CAD, we were not able to test the relationship in subjects with subclinical atherosclerosis. Fifth, the baseline examination in MPP did not report medications that influence autonomic function, such as opioids or sympathomimetics. Finally, women were highly underrepresented in MPP, meaning that we were underpowered for studying sex-specific relations.

In conclusion, we observed that subtle signs of cardiovascular autonomic dysfunction are associated with impaired lung function and may predict development of COPD in middle-aged subjects without airflow obstruction. This association is independent of the relationship between cardiovascular autonomic dysfunction and CAD. We propose that cardiovascular autonomic dysfunction should be taken into account when evaluating risk of future COPD, in addition to the currently known risk factors.

\section{References}

1 Curkendall SM, DeLuise C, Jones JK, et al. Cardiovascular disease in patients with chronic obstructive pulmonary disease, Saskatchewan Canada: cardiovascular disease in COPD patients. Ann Epidemiol 2006; 16: 63-70.

2 Feary JR, Rodrigues LC, Smith CJ, et al. Prevalence of major comorbidities in subjects with COPD and incidence of myocardial infarction and stroke: a comprehensive analysis using data from primary care. Thorax 2010; 65: 956-962.

3 Hole DJ, Watt GC, Davey-Smith G, et al. Impaired lung function and mortality risk in men and women: findings from the Renfrew and Paisley prospective population study. BMJ 1996; 313: 711-715; discussion 715-6.

4 Lange P, Nyboe J, Appleyard M, et al. Spirometric findings and mortality in never-smokers. J Clin Epidemiol 1990; 43: 867-873.

5 Underner M, Perriot J, Peiffer G. Sevrage tabagique du fumeur atteint de bronchopneumopathie chronique obstructive [Smoking cessation in smokers with chronic obstructive pulmonary disease]. Rev Mal Respir 2014; 31: 937-960.

6 Jimenez-Ruiz CA, Andreas S, Lewis KE, et al. Statement on smoking cessation in COPD and other pulmonary diseases and in smokers with comorbidities who find it difficult to quit. Eur Respir J 2015; 46: 61-79.

7 Anthonisen NR, Connett JE, Murray RP. Smoking and lung function of Lung Health Study participants after 11 years. Am J Respir Crit Care Med 2002; 166: 675-679.

8 Anthonisen NR, Connett JE, Enright PL, et al. Hospitalizations and mortality in the Lung Health Study. Am J Respir Crit Care Med 2002; 166: 333-339.

9 Hagstad S, Backman H, Bjerg A, et al. Prevalence and risk factors of COPD among never-smokers in two areas of Sweden - occupational exposure to gas, dust or fumes is an important risk factor. Respir Med 2015; 109: $1439-1445$.

10 Po JY, FitzGerald JM, Carlsten C. Respiratory disease associated with solid biomass fuel exposure in rural women and children: systematic review and meta-analysis. Thorax 2011; 66: 232-239.

11 Diaz-Guzman E, Aryal S, Mannino DM. Occupational chronic obstructive pulmonary disease: an update. Clin Chest Med 2012; 33: 625-636.

12 Kaufman JD, Adar SD, Barr RG, et al. Association between air pollution and coronary artery calcification within six metropolitan areas in the USA (the Multi-Ethnic Study of Atherosclerosis and Air Pollution): a longitudinal cohort study. Lancet 2016; 388: 696-704.

13 Roswall N, Raaschou-Nielsen $\mathrm{O}$, Ketzel M, et al. Long-term residential road traffic noise and $\mathrm{NO}_{2}$ exposure in relation to risk of incident myocardial infarction - a Danish cohort study. Environ Res 2017; 156: 80-86.

14 Sin DD, Man SF. Why are patients with chronic obstructive pulmonary disease at increased risk of cardiovascular diseases? The potential role of systemic inflammation in chronic obstructive pulmonary disease. Circulation 2003; 107: 1514-1519.

15 van Eeden SF, Sin DD. Chronic obstructive pulmonary disease: a chronic systemic inflammatory disease. Respiration 2008; 75: 224-238.

16 Sabater-Lleal M, Malarstig A, Folkersen L, et al. Common genetic determinants of lung function, subclinical atherosclerosis and risk of coronary artery disease. PLoS One 2014; 9: e104082.

17 Lamprecht B, McBurnie MA, Vollmer WM, et al. COPD in never smokers: results from the population-based burden of obstructive lung disease study. Chest 2011; 139: 752-763. 
18 Jensen MT, Suadicani P, Hein HO, et al. Elevated resting heart rate, physical fitness and all-cause mortality: a 16-year follow-up in the Copenhagen Male Study. Heart 2013; 99: 882-887.

19 Ricci F, Fedorowski A, Radico F, et al. Cardiovascular morbidity and mortality related to orthostatic hypotension: a meta-analysis of prospective observational studies. Eur Heart J 2015; 36: 1609-1617.

20 Fedorowski A, Hedblad B, Melander O. Early postural blood pressure response and cause-specific mortality among middle-aged adults. Eur J Epidemiol 2011; 26: 537-546.

21 van Gestel AJ, Steier J. Autonomic dysfunction in patients with chronic obstructive pulmonary disease (COPD). J Thorac Dis 2010; 2: 215-222.

22 Zaigham S, Nilsson PM, Wollmer P, et al. The temporal relationship between poor lung function and the risk of diabetes. BMC Pulm Med 2016; 16: 75.

23 Trell E. Community-based preventive medical department for individual risk factor assessment and intervention in an urban population. Prev Med 1983; 12: 397-402.

24 Quanjer PH, Stanojevic S, Cole TJ, et al. Multi-ethnic reference values for spirometry for the 3-95-yr age range: the global lung function 2012 equations. Eur Respir J 2012; 40: 1324-1343.

25 The definition of orthostatic hypotension, pure autonomic failure, and multiple system atrophy. J Auton Nerv Syst 1996; 58: 123-124.

26 Alberti KG, Zimmet PZ. Definition, diagnosis and classification of diabetes mellitus and its complications. Part 1: diagnosis and classification of diabetes mellitus provisional report of a WHO consultation. Diabet Med 1998; 15: 539-553.

27 Engstrom G, Segelstorm N, Ekberg-Aronsson M, et al. Plasma markers of inflammation and incidence of hospitalisations for COPD: results from a population-based cohort study. Thorax 2009; 64: 211-215.

28 Inghammar M, Engstrom G, Lofdahl CG, et al. Validation of a COPD diagnosis from the Swedish Inpatient Registry. Scand J Public Health 2012; 40: 773-776.

29 Tracey KJ. The inflammatory reflex. Nature 2002; 420: 853-859.

30 Chhabra SK, De S. Cardiovascular autonomic neuropathy in chronic obstructive pulmonary disease. Respir Med 2005; 99: 126-133.

31 Goulart CD, Cabiddu R, Schneiders PB, et al. Is cardiac autonomic modulation during upper limb isometric contraction and Valsalva maneuver impaired in COPD patients? Int J Chron Obstruct Pulmon Dis 2017; 12 : 849-857.

32 Lawlor DA, Patel R, Ebrahim S. Association between falls in elderly women and chronic diseases and drug use: cross sectional study. BMJ 2003; 327: 712-717.

33 Roig M, Eng JJ, Road JD, et al. Falls in patients with chronic obstructive pulmonary disease: a call for further research. Respir Med 2009; 103: 1257-1269.

34 Robertson D, DesJardin JA, Lichtenstein MJ. Distribution and observed associations of orthostatic blood pressure changes in elderly general medicine outpatients. Am J Med Sci 1998; 315: 287-295.

35 Walter RE, Beiser A, Givelber RJ, et al. Association between glycemic state and lung function: the Framingham Heart Study. Am J Respir Crit Care Med 2003; 167: 911-916.

36 Yeh HC, Punjabi NM, Wang NY, et al. Cross-sectional and prospective study of lung function in adults with type 2 diabetes: the Atherosclerosis Risk in Communities (ARIC) study. Diabetes Care 2008; 31: 741-746.

37 Pellegrino R, Viegi G, Brusasco V, et al. Interpretative strategies for lung function tests. Eur Respir J 2005; 26 : 948-968.

38 Fedorowski A, Hamrefors V, Sutton R, et al. Do we need to evaluate diastolic blood pressure in patients with suspected orthostatic hypotension? Clin Auton Res 2017; 27: 167-173.

39 Smith JJ, Porth CM, Erickson M. Hemodynamic response to the upright posture. J Clin Pharmacol 1994; 34: 375-386.

40 Stewart AG, Marsh F, Waterhouse JC, et al. Autonomic nerve dysfunction in COPD as assessed by the acetylcholine sweat-spot test. Eur Respir J 1994; 7: 1090-1095.

41 Baker JG, Wilcox RG. beta-Blockers, heart disease and COPD: current controversies and uncertainties. Thorax 2017; 72: 271-276.

42 Stewart AG, Waterhouse JC, Howard P. Cardiovascular autonomic nerve function in patients with hypoxaemic chronic obstructive pulmonary disease. Eur Respir J 1991; 4: 1207-1214.

43 Novak P. Orthostatic cerebral hypoperfusion syndrome. Front Aging Neurosci 2016; 8: 22.

44 Novak P. Quantitative autonomic testing. J Vis Exp 2011; 53: pii: 2502.

45 Engstrom G, Melander O, Hedblad B. Population-based study of lung function and incidence of heart failure hospitalisations. Thorax 2010; 65: 633-638. 